Research Article

\title{
Comparative Analysis of Low-Rank Matrix Denoising Algorithm-Based MRI and CT Images in Diagnosis of Cerebral Aneurysms
}

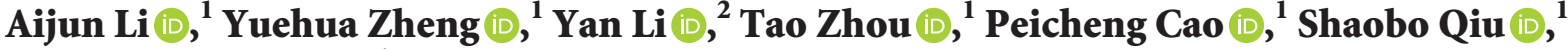 \\ and Jinpeng Wang $\mathbb{1}^{1}$ \\ ${ }^{1}$ Department of Neurosurgery, Weifang People's Hospital Shandong Province, Weifang 261021, China \\ ${ }^{2}$ Department of Outpatient Department, Weifang People's Hospital Shandong Province, Weifang 261021, China
}

Correspondence should be addressed to Jinpeng Wang; 170404106564@bitzh.edu.cn

Received 13 May 2021; Accepted 7 July 2021; Published 15 July 2021

Academic Editor: Gustavo Ramirez

Copyright (C) 2021 Aijun Li et al. This is an open access article distributed under the Creative Commons Attribution License, which permits unrestricted use, distribution, and reproduction in any medium, provided the original work is properly cited.

\begin{abstract}
This study aimed to compare the role of magnetic resonance imaging (MRI) and computed tomography (CT) images based on the low-rank matrix (LRM) denoising (LRMD) algorithm in the diagnosis of cerebral aneurysms (CAs). By comparing the role of MRI and $\mathrm{CT}$ in the diagnosis of CA, it would be helpful to formulate more reasonable diagnosis strategies and provide a solid foundation for clinical treatment of patients. 80 patients with cerebral aneurysm admitted to hospital were selected as the research objects. First, the LRMD algorithm was established and applied to the image denoising process of MRI and CT. Then, the diagnosis rate of CA by MRI and CT before and after denoising was compared, and the diagnostic rates of the two methods for aneurysms of different sizes were compared. Finally, the location, foci, and patient satisfaction of the aneurysm were compared. The results showed that the MRI and CT images after denoising with LRM were clearer, and the secondary structures in the brain were more obvious. It meant that LRMD had good image denoising effect. The diagnostic rate of denoised MRI and CT was improved. Although the difference was not statistically notable, the diagnostic rate of CT was obviously higher in contrast to MRI $(P<0.05)$. The diagnostic rate of CT for smaller aneurysms $(<3 \mathrm{~mm}$ and $3-5 \mathrm{~mm})$ was also notably higher in contrast to MRI $(P<0.05)$. However, there was no difference in the diagnosis of tumor location between the two. The clarity of CT diagnostic images was better than MRI $(P<0.05)$. Accordingly, patients were more satisfied with CT in contrast to MRI $(P<0.05)$. In summary, CT images based on the LRMD algorithm were superior to MRI in the diagnosis of CA, and it could provide more accurate diagnosis results.
\end{abstract}

\section{Introduction}

CA is an aneurysm-like protrusion caused by the destruction of the elastic layer in the wall of the intracranial artery, which results in the abnormal expansion of the lumen [1]. The cause of the disease is mostly congenital dysplasia, and others are disease interference factors, including hypertension and arteriosclerosis. Cerebrovascular diseases often occur in the middle-aged and elderly people. It is a common neurological disease with a high fatality rate and disability rate. Therefore, timely diagnosis and treatment are of great significance to patients $[2,3]$. MRI and CT technology are two commonly used clinical detection techniques, which include the detection of CA. MRI and CT detection have the characteristics of noninvasive, fast, and accurate. Therefore, they have gradually replaced the digital subtraction angiography technique, which is the standard procedure for the diagnosis and detection of CA [4].

The major limitation of MRI and CT imaging in diagnosis is the image quality. The various interferences encountered during image acquisition will cause noise in the image, resulting in deviation or even error in the final obtained information. Although the performance of equipment is constantly improving, software-based image 
denoising can be independent of equipment, with wide availability and high practicability. Image denoising algorithms can be divided into three categories: spatial domain denoising, transform domain denoising, and dictionary learning-based denoising [5-7]. Spatial domain denoising is divided into local filtering and nonlocal filtering. These processes mainly apply the similarity between image pixels or image blocks. The other two types of denoising project the image into a specific space, where the transform coefficients are filtered, and the processed coefficients are inversely transformed. Then, they put it back into the original space to complete the denoising [8]. Low-rank approximation and low-rank representation have great advantages in signal approximation and subspace segmentation and thus attract the attention of scholars. LRMD has strong stability and is widely used in artificial intelligence, image processing, and other fields. The main principle is performing low-rank decomposition of similar blocks to remove the noise in the image $[9,10]$. Generally speaking, there are more similar parts in the image, so the correlation of each row or column of the image matrix is relatively high. The rank of the matrix is much smaller than the number of rows and columns of the matrix, so the image matrix itself has low rank. The noise in the image will destroy the correlation of the image matrix and increase the rank of the image matrix. Therefore, the low-rank matrix denoising algorithm achieves the purpose of denoising by restricting the rank of the image matrix.

In summary, the LRMD algorithm was applied to MRI and CT images to improve the quality of images and provide better basis for clinical diagnosis. Based on that, the effects of MRI and CT were compared in the diagnosis of CA.

\section{Materials and Methods}

2.1. Establishment and Adoption of LRMD Algorithm. LRM restoration refers to a method that automatically recognizes the damaged elements and restores the original matrix when some elements of the matrix are severely damaged. The premise of this algorithm is that the original matrix is low rank or approximately low rank. It is assumed that the matrix $A$ is a LRM $M$ which is destroyed by the noise matrix $P$, and $P$ is a sparse matrix, and then low-rank sparse matrix factorization can be adopted to solve the problem. The LRM recovery is described by the following optimization:

$$
\begin{aligned}
& \min \operatorname{rank}(\mathrm{M})+\lambda\|P\|_{0}, \\
& \text { s.t. } \quad M+P=A .
\end{aligned}
$$

In (1), $\|P\|_{O}$ is the $M_{0}$ norm of the sparse matrix, that is, the number of nonzero elements in the matrix and $\lambda$ is the weight function. It takes a series of changes to turn an equation with multiple solutions into an equation that has a unique solution and can be solved.

Similar images have similar image structures. In the absence of pollution and defects, these similar structures should be in low-dimensional subspaces. A matrix composed of similar structures according to the same rule has a lower rank. When the image is contaminated or partially defective, the combined matrix will also be affected. These effects can be eliminated by LRM restoration processing. The matrix constructed by similar blocks after the noisy image is matched can be expressed as follows:

$$
A=M+P \text {. }
$$

In (2), $M$ is the LRM of pure similar blocks to be restored and $P$ is the sparse noise matrix. Therefore, the image denoising is transformed into a LRM restoration problem. After the similarity block matrix $A$ with noise is recovered by the LRM, the denoised similar block matrix $M$ is obtained. $M$ is put back to the position of the similar block correspondingly, and the denoised image will be obtained.

The denoising algorithm is made into an application and installed on the computer connected to MRI and CT. The image obtained from the instrument is preprocessed to make the image clearer, and then the doctor's diagnosis will be more efficient and accurate.

2.2. Clinical Data. A total of 80 patients with cerebral aneurysm admitted to the hospital from September 2018 to May 2020 were selected. All patients were diagnosed with CA by digital silhouette angiography (DSA) or surgery. There were 38 males and 42 females aged from 38 to 70 with an average age of 51. The clinical features of the patient were dizziness, headache, confusion, vomiting, and so on. Inclusion criteria are as follows: patients with cerebral aneurysm; patients went through the standard examination of the hospital; and patients were informed and voluntarily join the research. Exclusion criteria are as follows: patients with severe mental illness; patients with serious liver or kidney disease; and patients with incomplete information. All patients had signed informed consent forms, and the experiment was approved by the Ethics Committee of the hospital.

\subsection{Comparison of MRI and CT in the Diagnosis of Cerebral} Aneurysm. All patients received MRI and CT examinations. The examination was carried out uniformly. All patients were determined with CA by DSA or surgical examination.

MRI examination: the instrument was a superconducting magnetic resonance instrument produced in Japan. The relevant parameters were set as T1W1: TR/ $\mathrm{TE}=760 \mathrm{~ms} / 14 \mathrm{~ms}$ and $2000 / 47 \mathrm{~ms}$ and T2W1: TR/ $\mathrm{TE}=3900 \mathrm{~ms} / 102 \mathrm{~ms}$ and $2000 / 100 \mathrm{~ms}$. The scanning range was from the intracranial segment of the internal carotid artery to above the marginal corpus artery, and an image was acquired every $19^{\circ}$.

CT inspection: the instrument was a 128-slice spiral CT machine produced by Siemens, Germany. The scanning parameters were set as follows: layer thickness of $1.25 \mathrm{~mm}, 8$ layers per scan, tube voltage and tube current were $120 \mathrm{KV}$ and $330 \mathrm{~mA}$, pitch was 0.625 , matrix was $512 \times 512$, and rate was $0.5 \mathrm{~s} /$ turn. A high-pressure syringe was used to inject $80 \mathrm{~mL}$ of contrast agent at an injection rate of $3.5 \mathrm{~mL} / \mathrm{s}$ and a delay of 20 second.

All obtained patient images were denoised by LRM, and the diagnostic effects of MRI and CT on CA before and after image denoising were compared. Other aspects of the 
experiment included the rate of diagnosis, the rate of diagnosis for tumors of different sizes, the location of the tumor, the clarity of the lesion, and patient satisfaction. The rate of diagnosis was the ratio of the number of aneurysms detected to the total number of patients. The tumor size was divided into four grades: less than $3 \mathrm{~mm}, 3-5 \mathrm{~mm}, 5-10 \mathrm{~mm}$, and more than $10 \mathrm{~mm}$, and the number of confirmed patients at each grade was determined, respectively. The tumor location referred to the distribution of the tumor in different intracranial locations. The sharpness of the lesion was compared between MRI and CT images for intracranial imaging. A simple questionnaire survey was adopted to investigate patients' satisfaction with the examination, which was divided into three grades: satisfied, general satisfied, and dissatisfied.

2.4. Statistical Analysis. SPSS22.0 statistical software was adopted for data analysis. The obtained count data were represented by percentage, and the chi-square test was adopted to compare the probabilities between the two groups. $P<0.05$ was considered statistically significant. Origin 9.0 was adopted to draw the images.

\section{Results}

3.1. Image Optimization. Figures 1 and 2 show that the obtained aneurysm images were denoised by LRM. Figure 1(a) shows the CT before treatment, while Figure 1(b) shows the CT after treatment. Figure 2(a) is the MRI image before denoising, while Figure 2(b) is the MRI image after denoising. The images after noise reduction were clearer, the brain secondary structure was more distinct, and the lesion exposure was more obvious.

3.2. Contrast of the Diagnosis Rate of CA by Two Diagnostic Methods. In Figure 3, in the diagnosis of 80 patients, the diagnostic rate of CT for CA (about 100\%) was remarkably higher in contrast to MRI (about $85 \%$ ), with notable difference and $P<0.05$.

\subsection{Contrast of the Diagnosis Rate of CA by MRI and CT before} and after Image Denoising. The quality of the image affected the diagnosis result to a certain extent. In Figure 4, after image denoising, the diagnosis rate of CA by CT and MRI has been improved, but this difference was not particularly notable $(P>0.05)$.

3.4. Contrast of the Diagnosis Rate of Different Sizes of CA by Two Diagnostic Methods. In Table 1 and Figure 5, CT and MRI both had lower detection rates for tumors with a smaller diameter (less than $5 \mathrm{~mm}$ ) than those with a larger diameter (more than $5 \mathrm{~mm}$ ). In tumors with a smaller diameter (less than $5 \mathrm{~mm}$ ), the detection rate of CT was higher in contrast to MRI, and the difference was notable $(P<0.05)$. In tumors with a larger diameter (more than $5 \mathrm{~mm}$ ), there was no particularly notable difference in the detection rate of the two methods $(P>0.05)$.
3.5. CT and MRI Diagnosis of Cerebral Aneurysm Location. Table 2 shows the results of CT and MRI diagnosis of the location of CA. The incidence in aneurysms in the middle cerebral artery, anterior artery, and posterior artery was higher in contrast to internal carotid artery, vertebral artery, and basilar artery. The results showed that there was no particularly obvious difference between CT and MRI in the diagnosis of tumor location $(P>0.05)$.

3.6. Contrast between CT and MRI for Focal Definition of Aneurysms. In Table 3, it compared the clarity of CT and MRI images in diagnosing aneurysms. The results showed that the clarity of CT images was particularly higher in contrast to MRI, and the unclarity of CT images was notably lower in contrast to MRI, and $P<0.05$.

3.7. Contrast of Patients' Satisfaction with CT and MRI Examinations. Table 4 shows patients' satisfaction with CT and MRI tests. The satisfaction of patients with CT examination was remarkably higher in contrast to MRI examination, and the dissatisfaction was remarkably lower than it, and $P<0.05$.

\section{Discussion}

The treatment of cerebral aneurysm is a very complicated process, and accurate diagnosis of the patient is a critical step [11]. The patient's diagnosis rate is closely related to the quality of the examination images. The quality of CT and MRI images processed by the LRMD algorithm had been improved, which provided further convenience for the diagnosis of patients. In the clinical experiment, the processed images could improve the diagnosis rate of $\mathrm{CA}$, but this change was not particularly notable and had no statistical significance. It may be because the accuracy of the detection instrument itself was relatively high, and the image quality was high, so it is not very dependent on the image denoising means.

DSA is often adopted in the detection of CA. However, since DSA has certain limitations in the diagnosis of the spatial location of aneurysms, the cost of diagnosis is expensive and it is a traumatic examination, which limit its clinical application and development [12]. Studies have shown that MRI could more accurately measure the size of aneurysms and clearly show the lesions at the base of the skull. The advantages of CT images and MRI in the detection of CA were also elegant. The study of Muthusami et al. [13] also proved that CT was better than MRI in the diagnosis of pediatric cerebrovascular diseases. The acceptance among patients was also higher, which was consistent with the findings of this study that the diagnosis rate of CT for patients was higher in contrast to MRI. Blokker et al. [14] pointed that cerebral aneurysm was a disease that was extremely prone to death and disability. The main clinical manifestation was subarachnoid hemorrhage. Effective diagnosis and timely treatment could help to reduce the patient's risk of death. However, the MRI scan time was longer, and the image quality would decrease due to the slight 

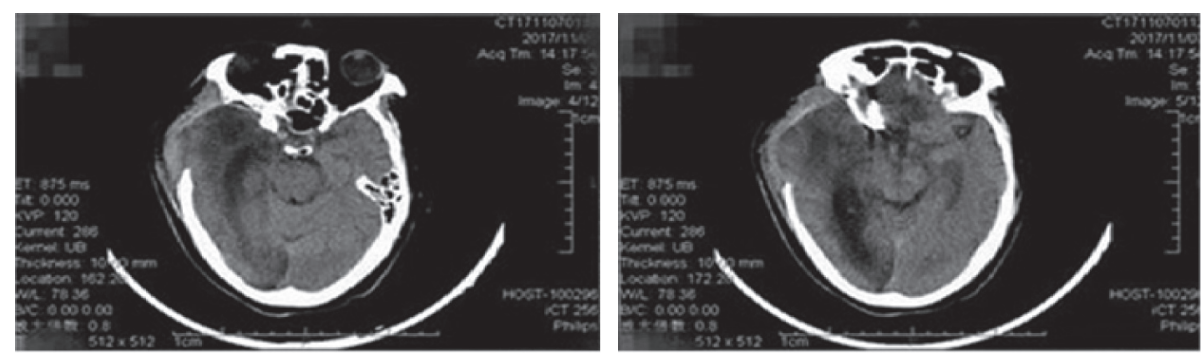

(a)
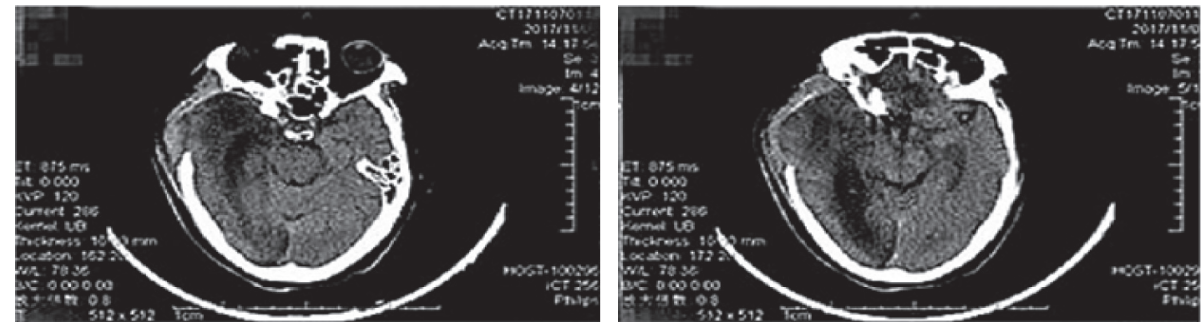

(b)

Figure 1: Contrast of CT images of cerebral aneurysm (a) before denoising and (b) after denoising by LRM.
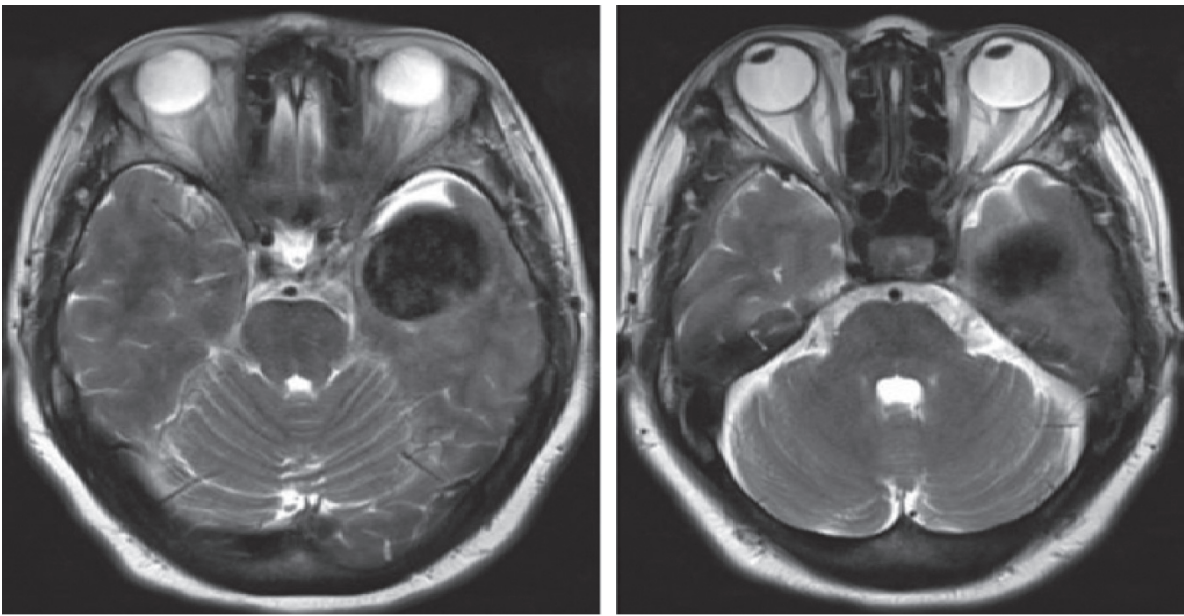

(a)
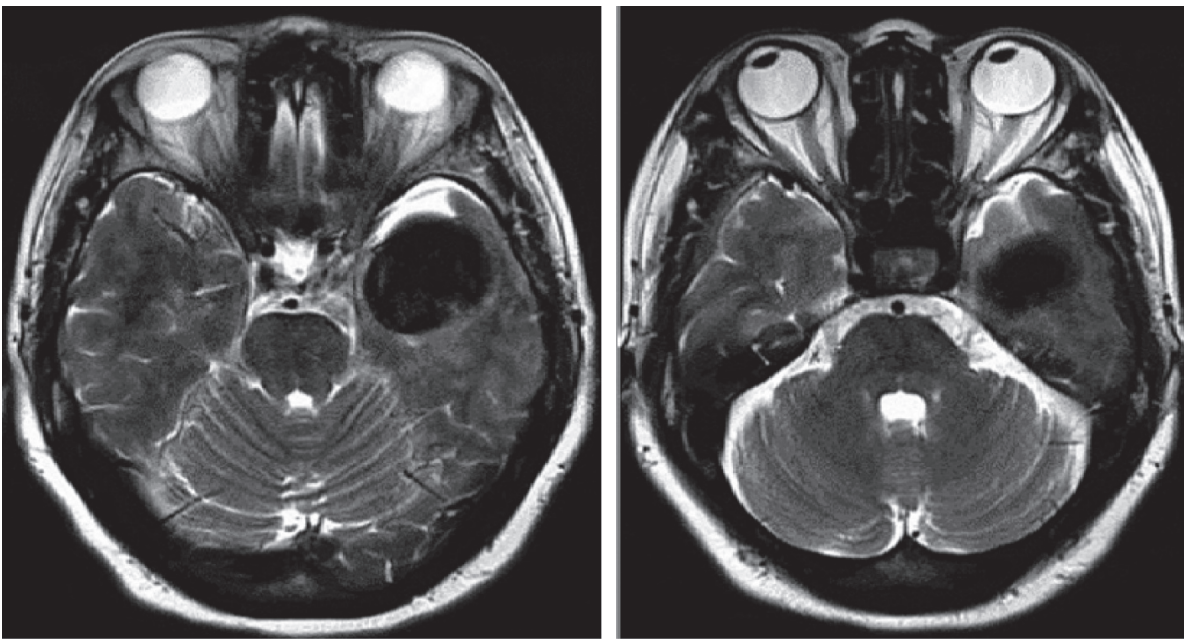

(b)

FIGURE 2: Comparison of MRI images of cerebral aneurysm (a) before denoising and (b) after denoising by LRM. 


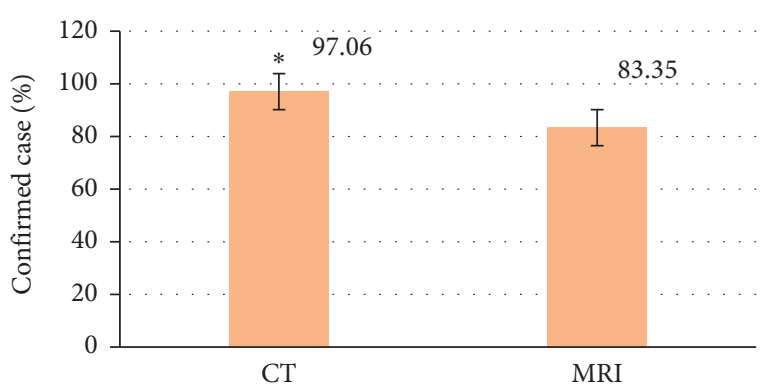

Figure 3: Contrast of the diagnosis rate of the two diagnostic methods. ${ }^{*}$ indicates that $P<0.05$, and the difference is statistically notable.

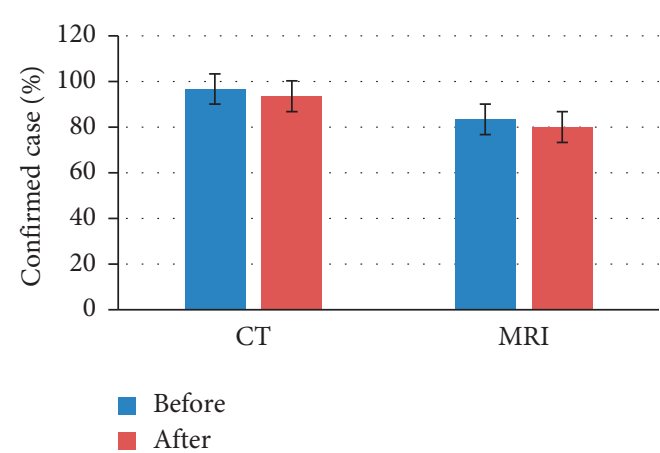

FIgURE 4: Contrast of CT and MRI diagnosis rates of CA before and after image denoising.

TABle 1: Contrast of the diagnosis rate of different sizes of CA by two diagnostic methods.

\begin{tabular}{lcccc}
\hline & CT & MRI & $\chi^{2}$ & $P$ \\
\hline$<3 \mathrm{~mm}(n=15)$ & $13(86.7 \%)$ & $8(53.3 \%)$ & 3.587 & $<0.05$ \\
$3-5 \mathrm{~mm}(n=28)$ & $26(92.8 \%)$ & $19(67.9 \%)$ & 3.127 & $<0.05$ \\
$5-10 \mathrm{~mm}(n=27)$ & $27(100.0 \%)$ & $27(100.0 \%)$ & - & - \\
$>10 \mathrm{~mm}(n=10)$ & $10(100.0 \%)$ & $10(100.0 \%)$ & - & - \\
\hline
\end{tabular}

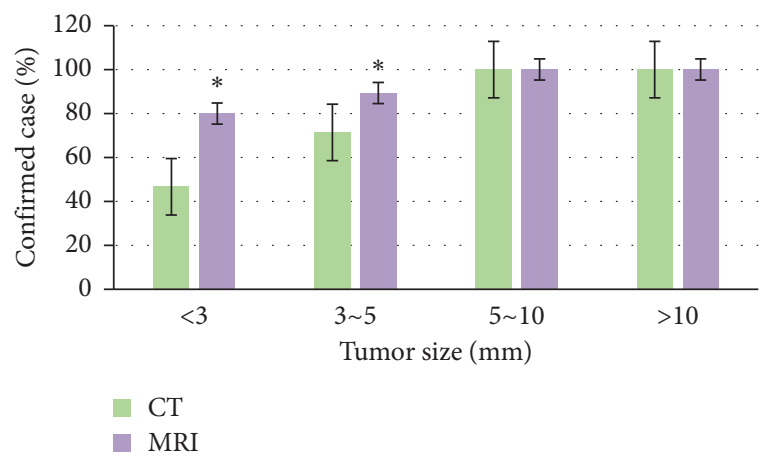

FIgURE 5: Contrast of the diagnostic rates of different sizes of CA by the two diagnostic methods. ${ }^{*}$ indicates that $P<0.05$, and the difference is statistically notable.

movement of the patient. Therefore, this examination was not suitable for patients with ruptured aneurysms and bleeding. Cho et al. [15] and Ikawa et al. [16] suggested that the lower detection rate of smaller tumors was also a
TABLE 2: Contrast of CT and MRI diagnosis results of cerebral aneurysm location.

\begin{tabular}{lcccc}
\hline Tumor location & CT & MRI & $\chi^{2}$ & $P$ \\
\hline Middle cerebral artery & $28(35.9 \%)$ & $21(31.3 \%)$ & 0.067 & 0.819 \\
Anterior cerebral artery & $21(26.9 \%)$ & $20(29.8 \%)$ & 0.023 & 0.870 \\
Posterior cerebral artery & $11(14.1 \%)$ & $10(14.9 \%)$ & 0.019 & 0.905 \\
Internal carotid artery & $8(10.3 \%)$ & $6(8.9 \%)$ & 0.058 & 0.836 \\
Vertebral artery & $5(6.4 \%)$ & $5(7.4 \%)$ & 0.002 & 0.962 \\
Basilar artery & $5(6.4 \%)$ & $5(7.4 \%)$ & 0.004 & 0.987 \\
Total & 78 & 67 & - & - \\
\hline
\end{tabular}

TABle 3: Contrast of CT and MRI in diagnosis of aneurysms.

\begin{tabular}{lcccc}
\hline & CT & MRI & $\chi^{2}$ & $P$ \\
\hline Clear & $72(90.0 \%)$ & $65(81.2 \%)$ & 3.765 & $<0.05$ \\
Unclear & $8(10.0 \%)$ & $15(18.8 \%)$ & 2.453 & $<0.05$ \\
Total & 80 & 80 & - & - \\
\hline
\end{tabular}

TABLe 4: Patients' satisfaction with CT and MRI examinations.

\begin{tabular}{lcccc}
\hline & Satisfied & General satisfied & Dissatisfied & Total \\
\hline MRI & $55(68.8 \%)$ & $15(18.8 \%)$ & $10(12.5 \%)$ & 80 \\
CT & $68(85 \%)$ & $7(8.8 \%)$ & $5(6.3 \%)$ & 80 \\
$\chi^{2}$ & 3.238 & 2.698 & 2.034 & - \\
$P$ & $<0.05$ & $<0.05$ & $<0.05$ & - \\
\hline
\end{tabular}

drawback of MRI detection, which was consistent with this finding that CT had a higher detection rate for smaller tumors than MRI $(P<0.05)$. As a noninvasive examination, the application value of CT has been fully affirmed. It has gradually become a routine examination method for clinical diagnosis of CA. In contrast to MRI, CT examination time was short, and the image had three-dimensional characteristics. It could clearly show the spatial relationship between aneurysms and adjacent tissues, which was conducive to the formulation of surgical plans $[17,18]$. In addition, it was found that the sharpness of the lesion in CT examination was also better in contrast to MRI $(P<0.05)$, and the patients' satisfaction with CT examination was also higher in contrast to MRI $(P<0.05)$. These results indicated that the diagnostic effect of $\mathrm{CT}$ was obviously better in contrast to MRI.

\section{Conclusion}

It analyzed and compared the role of MRI and CT images based on the LRMD algorithm in the diagnosis of CA. The results showed that CT imaging was superior to MRI imaging in the diagnosis of CA in all aspects, especially in the diagnosis rate, the detection rate of tumor size, the definition of the lesion, and the degree of patient satisfaction. The influence of low-rank denoising on the diagnosis result was not very notable. CT imaging could be taken as an effective means to diagnose the occurrence of aneurysms. However, due to the limited sample of this research and the single research method, it cannot be concluded that image denoising is not an effective step in the diagnosis of aneurysm. On the contrary, research efforts should be 
increased to further verify the role of image denoising in the diagnosis of aneurysm. By comparing the effects of MRI and CT in the diagnosis of CA, the advantages of CT in the diagnosis of aneurysms are highlighted, which will help improve the accuracy and efficiency of clinical diagnosis of CA. [19].

\section{Data Availability}

No data were used to support this study.

\section{Conflicts of Interest}

The authors declare that they have no conflicts of interest.

\section{Authors' Contributions}

Aijun Li and Yuehua Zheng contributed equally to this work.

\section{Acknowledgments}

This work was supported by Shandong Medical and Health Science and Technology Development Plan (project no. 2017WS248) and Weifang Science and Technology Development Plan (project no. 2019YX041).

\section{References}

[1] J. E. Park, S. C. Jung, S. H. Lee et al., "Comparison of 3D magnetic resonance imaging and digital subtraction angiography for intracranial artery stenosis," European Radiology, vol. 27, no. 11, pp. 4737-4746, 2017.

[2] J. Qin, X. Shen, H. Chen et al., "A fusion algorithm for medical structural and functional images based on adaptive image decomposition," Multimedia Tools and Applications, vol. 78, no. 22, Article ID 32605, 2019.

[3] S. A. Nabavizadeh, A. Vossough, R. N. Ichord et al., "Intracranial aneurysms in sickle cell anemia: clinical and imaging findings," Journal of Neuro Interventional Surgery, vol. 8, no. 4, pp. 434-440, 2016.

[4] L. R. M. Menchón, W. F. Simmross, D. L. H. P. Casaseca et al., "Reconstruction techniques for cardiac cine MRI," Insights into imaging, vol. 10, no. 1, 2019.

[5] R. Razaghi, H. Biglari, and A. Karimi, "Risk of rupture of the cerebral aneurysm in relation to traumatic brain injury using a patient-specific fluid-structure interaction model," Computer Methods and Programs in Biomedicine, vol. 176, pp. 9-16, 2019.

[6] G. B. Toth, C. G. Varallyay, A. Horvath et al., "Current and potential imaging applications of ferumoxytol for magnetic resonance imaging," Kidney International, vol. 92, no. 1, pp. 47-66, 2017.

[7] N. J. Lee, M. S. Chung, S. C. Jung et al., "Comparison of highresolution MR imaging and digital subtraction angiography for the characterization and diagnosis of intracranial artery disease," American Journal of Neuroradiology, vol. 37, no. 12, pp. 2245-2250, 2016.

[8] I. L. Maier, J. R. Leyhe, I. Tsogkas et al., "Diagnosing early ischemic changes with the latest-generation flat detector CT: a comparative study with multidetector CT," American Journal of Neuroradiology, vol. 39, no. 5, pp. 881-886, 2018.
[9] K. Guggenberger, A. J. Krafft, U. Ludwig et al., "High-resolution compressed-sensing T1 black-blood MRI," Clinical Neuroradiology, vol. 31, no. 1, pp. 1-10, 2019.

[10] C. Zhu, X. Wang, L. Eisenmenger et al., "Surveillance of unruptured intracranial saccular aneurysms using noncontrast 3D-black-blood MRI: comparison of 3D-TOF and contrast-enhanced MRA with 3D-DSA," American Journal of Neuroradiology, vol. 40, no. 6, pp. 960-966, 2019.

[11] N. Shono, T. Kin, S. Nomura et al., "Microsurgery simulator of cerebral aneurysm clipping with interactive cerebral deformation featuring a virtual arachnoid," Operative Neurosurgery, vol. 14, no. 5, pp. 579-589, 2018.

[12] J. R. Anderson, W. L. Thompson, A. K. Alkattan et al., "Threedimensional printing of anatomically accurate, patient specific intracranial aneurysm models," Journal of Neurointerventional Surgery, vol. 8, no. 5, pp. 517-520, 2016.

[13] P. Muthusami, N. Shkumat, V. Rea et al., "CT reconstruction and MRI fusion of 3D rotational angiography in the evaluation of pediatric cerebrovascular lesions," Neuroradiology, vol. 59, no. 6, pp. 625-633, 2017.

[14] B. M. Blokker, A. C. Weustink, I. M. Wagensveld et al., "Conventional autopsy versus minimally invasive autopsy with postmortem MRI, CT, and CT-guided biopsy: comparison of diagnostic performance," Radiology, vol. 289, no. 3, pp. 658-667, 2018.

[15] S. Cho, J. Lee, K. Ryu et al., "Diagnosis of cerebral aneurysm via magnetic resonance angiography screening: emphasis on legal responsibility increases false positive rate," Neurointervention, vol. 13, no. 1, p. 48, 2018.

[16] F. Ikawa, A. Morita, S. Tominari et al., "Rupture risk of small unruptured CA," Journal of Neurosurgery, vol. 132, no. 1, pp. 69-78, 2019.

[17] R. P. Martínez, S. P. Lownie, and D. Pelz, "Intra-arterial use of abciximab in thromboembolic complications associated with cerebral aneurysm coiling: the london ontario experience," World Neurosurgery, vol. 100, pp. 342-350, 2017.

[18] S. Winklhofer, R. Hinzpeter, D. Stocker et al., "Combining monoenergetic extrapolations from dual-energy CT with iterative reconstructions: reduction of coil and clip artifacts from intracranial aneurysm therapy," Neuroradiology, vol. 60, no. 3, pp. 281-291, 2018.

[19] N. Turan, R. A. Heider, A. K. Roy et al., "Current perspectives in imaging modalities for the assessment of unruptured intracranial aneurysms: a comparative analysis and review," World Neurosurgery, vol. 113, pp. 280-292, 2018. 